\title{
Investissement militant et violence contre soi au sein du Parti des travailleurs du Kurdistan
}

\section{Olivier Grojean}

\section{(2) OpenEdition \\ 1 Journals}

\section{Édition électronique}

URL : http://journals.openedition.org/conflits/2108

DOI : $10.4000 /$ conflits. 2108

ISSN : $1777-5345$

\section{Éditeur :}

CCLS - Centre d'études sur les conflits lilberté et sécurité, L'Harmattan

\section{Édition imprimée}

Date de publication : 1 novembre 2006

Pagination : 101-112

ISBN : 2-296-01436-4

ISSN : 1157-996X

\section{Référence électronique}

Olivier Grojean, « Investissement militant et violence contre soi au sein du Parti des travailleurs du Kurdistan », Cultures \& Conflits [En ligne], 63 | automne 2006, mis en ligne le 01 décembre 2006, consulté le 30 mars 2021. URL : http://journals.openedition.org/conflits/2108 ; DOI : https://doi.org/ $10.4000 /$ conflits. 2108

Ce document a été généré automatiquement le 30 mars 2021.

Creative Commons License 


\title{
Investissement militant et violence contre soi au sein du Parti des travailleurs du Kurdistan
}

\author{
Olivier Grojean
}

1 A la croisée de plusieurs domaines de recherche (ethnologie, psychologie sociale, sociologie des mobilisations, des conflits et de la violence, sociologie des religions), les études sur les formes d'action sacrificielle pour une cause politique se sont multipliées depuis 2001. Le décloisonnement disciplinaire qui s'opère au sein de ce champ de recherche en formation a d'ores et déjà permis de confronter les points de vue, de faire varier les échelles et les focales d'analyse et de dépasser certaines difficultés méthodologiques inhérentes à ce type d'étude (difficultés d'accès au terrain, « sensibilité » du sujet et surtout complexité du traitement des données recueillies). La singularité des modes d'action dits « suicides » conduit toutefois la plupart des auteurs à envisager leur objet de recherche au sein de deux familles de faits sociaux spécifiques: les techniques de guerre et la mort volontaire. Ces deux catégories fusionnent d'ailleurs dans les principaux qualificatifs utilisés pour décrire ces actions (attaque ou attentat-suicide, action kamikaze, etc.). Cette conceptualisation, souvent implicite, se fonde évidemment sur une certaine réalité, empiriquement incontestable ; elle ne nous semble pas cependant épuiser les manières d'envisager ces phénomènes.

2 Au-delà des définitions étiologiques ou téléologiques du phénomène de martyre, il peut en effet sembler judicieux d'axer la réflexion sur les formes de violence contre soi pour une cause politique. Cette construction de l'objet, volontairement large, part d'une hypothèse jusqu'à présent peu explorée : la relative continuité entre les engagements qui requièrent toujours la mort de l'activiste (les violences dites « auto-sacrificielles ») et ceux qui impliquent des blessures ou des lésions non nécessairement mortelles (les violences "auto-infligées»). Deux raisons peuvent être avancées pour justifier ce choix. Certaines formes de violence qu'on pourrait a priori qualifier de «sacrificielles » tendent d'abord à s'approcher de la mort sans pour autant toujours l'atteindre. Ainsi, sur 164 militants ou sympathisants du Parti des travailleurs du Kurdistan (Partiya 
Karkerên Kurdistan, PKK) s'étant immolés par le feu entre 1982 et 2006, moins de la moitié est effectivement décédée $(46,7 \%)^{1}$. Inversement, les grèves de la faim - souvent distinguées des violences sacrificielles ${ }^{2}$ - peuvent parfois prendre la forme de véritables «jeûnes de la mort» et faire des dizaines de victimes, comme lors du mouvement contre la réforme des prisons en Turquie depuis 2000. Il semble alors nécessaire de ne pas considérer la mort comme une variable dépendante dans la construction de notre objet de recherche. Une deuxième raison tient à l'utilisation fréquente de diverses formes de violence contre soi par des individus appartenant à un même groupe. Il n'est en effet pas rare que des sans-papiers en grève de la faim menacent de s'immoler par le feu, voire qu'ils passent réellement à l'acte ${ }^{3}$. De même, des groupes radicaux kurdes, turcs, palestiniens, iraniens ou tamouls ont eu recours, dans des proportions variées et à des moments différents, aux grèves de la faim et aux attaques-suicides. Tous ces exemples invitent in fine à ne pas séparer trop radicalement violences auto-sacrificielles et violences auto-infligées. Cette hypothèse ne signifie pourtant aucunement que nous assimilions un mode d'engagement " suicidaire » (c'està-dire risqué - voire très risqué - comme la grève de la faim) aux modes d'engagement "suicides" (qui paraissent supprimer l'idée même de risque); mais en déplaçant le regard du suicide vers les techniques de soi, des répertoires d'action des organisations vers les modalités d'investissement des militants, il s'agira d'abord de s'interroger plus généralement sur la construction sociale de l'aspiration à meurtrir voire à détruire son propre corps, de resituer les pratiques et les "arts de faire» qui constituent le quotidien de la lutte d'un groupe donné et surtout de prendre au sérieux les effets de la socialisation militante sur les individus engagés.

3 L'objectif de cet article sera donc d'analyser, à partir d'une sociologie de l'investissement militant, les processus individuels et collectifs qui peuvent conduire à se faire violence, en partant de l'hypothèse que les violences auto-sacrificielles, tout comme les violences auto-infligées, sont fonction d'un certain rapport à soi et à son environnement, construit socialement dans un contexte donné. Le cas kurde nous aidera ici à montrer comment se développe progressivement un certain imaginaire de soi et de son propre corps pouvant être mobilisé en situation par des acteurs ayant actualisé certaines dispositions. Après avoir brièvement observé comment le corps, devenu un enjeu fondamental au sein du conflit kurde, a pu être transformé en moyen de lutte, l'étude de la fabrique des martyrs permettra de saisir les liens qu'entretiennent idéal d'engagement et techniques de soi au sein du PKK. Sans proposer d'explication "achevée», il sera alors possible de dégager quelques pistes permettant de penser les rapports entre violences contre soi et contextes de l'engagement.

La fabrique de l'arme corporelle

4 Dans tout conflit armé, le corps est un des principaux sites de marquage de la violence et de la domination. Mais ce constat est sans doute encore plus flagrant dans les conflits de différenciation interne marqués par l'utilisation de méthodes non conventionnelles, comme la guerre de guérilla et la contre-insurrection. Hanté par le spectre du séparatisme et du "terrorisme ", l'Etat turc a dès le milieu des années 1980 mis en œuvre dans les régions kurdes des techniques contre-insurrectionnelles ayant pour objectif l'élimination du PKK par le contrôle de la population. L'ensemble de ces dispositifs a progressivement constitué l'équivalent d'un "régime scopique ${ }^{4}$ ", qui vise 
à rendre visible la sphère de l'intime tout en recouvrant d'un voile opaque toute activité relevant en principe des affaires publiques.

La criminalisation de la langue maternelle, la surveillance des activités quotidiennes ou la politisation et le viol des espaces domestiques, l'anonymat des manifestants brisé par les nombreuses caméras de la police, et, inversement, l'interdiction de la zone aux journalistes, la condamnation à la clandestinité de toute activité politique pro-kurde et le nombre élevé d'assassinats dont les auteurs sont restés " inconnus », ont participé à la mise en place de ce régime spécifique qui tente d'inverser sphère privée et sphère publique. Mais au niveau individuel, c'est sur le corps que s'est focalisé le contrôle de l'Etat et des forces paramilitaires. On peut ainsi mentionner pêle-mêle les tentatives de disciplinarisation du corps par l'école, l'utilisation systématique de la torture dans les commissariats, l'incarcération, les disparitions ou la non-remise des corps des guérilleros tués à leurs familles, les fouilles humiliantes aux checkpoints, les stratégies de repérage «au faciès» en fonction d'indices phonétiques (accent), pileux (les moustaches peuvent ainsi révéler l'appartenance politique ${ }^{5}$ ), ou morphologiques (être «basané ", être amputé d'un pied ou d'une main peuvent révéler une vie clandestine dans les montagnes)... A certains moments, et même si ces phénomènes ne sont pas propres aux situations contre-insurrectionnelles, la déshumanisation de l'ennemi a également engendré des formes d'humiliation ou de mutilation des corps. Ainsi, par exemple, lorsque des soldats turcs ont forcé des villageois à manger leurs propres excréments à Yesilyurt en janvier 1989, ou quand des militaires turcs ont posé, en mars ou avril 1995, devant les corps décapités et mutilés de quatre combattants du PKK ${ }^{6} \ldots$

6 Or, toutes ces pratiques constituent des moyens d'» objectivation politique des corps " susceptibles d'être réappropriés par des acteurs individuels ayant un accès limité aux autres formes de protestation ${ }^{7}$. Les grèves de la faim de militants emprisonnés en Turquie et, au-delà, de réfugiés sans papiers en Europe, très nombreuses, peuvent être lues de cette manière ${ }^{8}$. Exclusion de la communauté des citoyens, emprisonnement, humiliations, mauvais traitements, viols et/ou tortures, vécus directement ou médiatisés et "incorporés " à une histoire personnelle, sont autant d'empreintes synonymes de la négation de tout statut. En se soustrayant à l'emprise de l'Etat sur son propre corps, le gréviste s'inscrit dans une contestation du monopole de la violence légitime de l'Etat sur les corps ; en tentant de se réapproprier le contrôle de son propre corps, il réclame également un statut qui lui est dénié ${ }^{9}$. La perception commune de la grève de la faim comme mode d'action non violent semble ici trompeuse : l'étude des mobilisations kurdes en Europe tend en effet à montrer que le recours à la grève de la faim et le recours à la violence contre autrui apparaissent souvent de manière synchrone. Cette utilisation stratégique de la violence contre soi pourrait laisser penser d'autre part qu'il ne s'agit ici que d'une instrumentalisation rationnelle de sa propre culture, de ses propres stigmates. Mais les nombreuses autres formes plus spontanées de violence contre soi - les tentatives d'immolation par le feu de sans-papiers, ou encore le fait de se taper violemment la tête contre les murs ou les fenêtres d'un bus, comme ont pu le faire certains réfugiés kurdes d'Irak à Sangatte - montrent à quel point l'imaginaire du corps souffrant peut être mobilisé inconsciemment et sans préméditation dans l'action.

7 Les acteurs collectifs semblent tout autant concernés par ce phénomène. Fondé en 1978, le PKK s'est en effet construit au contact de l'imaginaire du corps souffrant et en interaction avec son environnement régional, marqué en 1975 par la défaite du 
mouvement de Barzani en Irak, la révolution iranienne et le coup d'Etat en Turquie en 1980. S'il adopte, comme quasiment toutes les autres organisations kurdes de l'époque, le marxisme-léninisme, il reste avant tout nationaliste et va trouver dans l'historiographie kurde des années 1920-1940 un certain nombre d'éléments lui permettant de justifier son combat. Inversant l'historiographie turque érigée en idéologie d'Etat après 1923, empreint de darwinisme social et d'anthropologie raciale ${ }^{10}$, se nourrissant de la répression des nombreuses révoltes kurdes des années 1920-1930, le nationalisme kurde se fonde en effet sur les idées de lutte pour la survie de la race ou de l'individu comme élément primordial de la nation biologique. Le corps kurde s'inscrit alors dans un système politique complexe. En tant qu'unité fondamentale de la nation, il est transcendé par sa beauté et son esprit. Mais en tant que participant de l'édifice global, il peut également être vérolé et contaminer ses membres, construisant en retour, comme dans l'historiographie turque, une thématique de l'unité et de l'ennemi intérieur.

8 Cette historiographie va prendre un sens particulier au contact de la répression des années 1970 et 1980 : le corps va alors devenir emblématique de la nation kurde. Le corps ethnicisé d'abord, avec ses vêtements traditionnels, ses danses et ses chants, si caractéristique des manifestations kurdes en Turquie et en Europe. Le corps torturé et supplicié ensuite, qui, affiché sur nombre de documents à vocation interne (journaux, revues, affiches placardées sur les murs des associations affiliées au PKK) ou externe (tracts, communiqués) ${ }^{11}$, permet tout autant de justifier la lutte que de dénoncer les persécuteurs ${ }^{12}$. Le corps violenté enfin, ultime recours contre l'arbitraire des prisons turques, et imposé aux occidentaux dans de nombreuses grèves de la faim de soutien en Europe dans les années 1980 et 1990. Dans tous les cas, le corps souffrant, ordinairement confiné à la sphère privée, semble ainsi être devenu une arme politique de dénonciation qui renverse le stigmate de la violence subie ${ }^{13}$. Comme si, en visant à rétablir la publicité de la blessure politique ${ }^{14}$, les acteurs tentaient de renverser l'ordre contre-insurrectionnel qui fragilise, voire détruit, la distinction entre ce qui est public et ce qui est privé. S'il est extrêmement difficile d'évaluer les conséquences d'un conflit sur la construction subjective de soi, il apparaît donc que le corps est devenu un « repère » et un enjeu important au sein du conflit. Contrôlé, supplicié et nié, il a été récupéré par un certain nombre d'acteurs, qui en ont fait un nouveau moyen de lutte.

Martyre, idéal d'engagement et techniques de soi

9 L'absence de statut ainsi que la violence subie - directement ou médiatisée apparaissent donc bien dans le cas kurde comme des éléments nécessaires à la fabrique de l'arme corporelle; elles n'expliquent pas cependant un recours aussi fréquent à la violence contre soi : des centaines de grèves de la faim collectives ont en effet été menées en Turquie et en Europe depuis 1980 et environ 200 militants ou sympathisants du PKK ont tenté de s'immoler par le feu ou se sont fait exploser lors d'opérations armées depuis 1982. La notion de "martyre ", centrale dans la socialisation militante au sein du parti, peut permettre d'aller plus loin. Trois catégories de martyrs (Sehit) peuvent traditionnellement être distinguées chez les Kurdes de Turquie : les victimes non combattantes, les combattants tués pour la cause et les martyrs «emblématiques 15 ». Dans l'imaginaire de la lutte, il existe une relation quasi organique entre ces trois catégories («en raison des nombreux innocents assassinés, les Kurdes ont pris les armes et se sont fait tuer en suivant l'exemple des héros morts pour le Kurdistan »), ce qui nécessite de ne pas les séparer de manière trop rigide. C'est la catégorie des martyrs 
"emblématiques» qui apparaît néanmoins fondamentale dans la construction d'un idéal d'engagement qui passe par la disciplinarisation du corps.

Débutée dans les années 1930, la «fabrique des martyrs » héroïques est un phénomène complexe qui obéit tout autant à des considérations stratégiques (volonté de mettre en valeur certains héros à des fins de mobilisation ${ }^{16}$ ) qu'à des processus davantage sociohistoriques, indépendants des acteurs nationalistes (tradition orale, mémoire locale des révoltes et de leur répression). Il serait pourtant bien difficile d'établir un rapport de causalité directe entre imaginaire des héros nationaux et violence contre soi, tout du moins durant les premières années du PKK. Les nationalistes kurdes qui s'engagent dans les années 1960-1970 redécouvrent certes ces premiers martyrs, éclipsés après la répression de la révolte de Dersim (1936-1938). Mais les fondateurs du PKK, fortement influencés par le marxisme-léninisme, semblent bien davantage mettre en avant les "structures objectives » de la domination turque, aux dépens des événements et des personnages héroïques ${ }^{17}$. Ce n'est en fait qu'après les morts violentes de la prison de Diyarbakir qu'une véritable martyrologie " partisane » se met en place.

11 Les violences auto-sacrificielles émergent en effet dans la prison $n^{\circ} 5$ de Diyarbakir, où les conditions de détention sont particulièrement dures. Une première grève de la faim est organisée en décembre 1980, suivie de plusieurs autres au début de l'année 1981. Au mois de mars est lancé un premier «jeûne de la mort » qui cesse au bout de 45 jours après l'acceptation de certaines revendications par la direction de la prison. Le 21 mars 1982, le suicide par pendaison du responsable de la propagande du PKK, Mazlum Dogan, marque une nouvelle étape: quatre immolations par le feu suivent le 18 mai puis quatre militants meurent au cours d'un « jeûne de la mort » en septembre de la même année (Mehmet Ayri Durmus et Kemal Pir - membres du Comité central - ainsi que Akif Yilmaz et Ali Çiçek). Les premières formes de violence contre soi apparaissent donc en prison et sont fortement conditionnées par cet environnement; la diffusion de ces pratiques à l'extérieur de l'univers carcéral s'opère en 1988 avec l'immolation par le feu de trois mères de détenus qui protestent contre les conditions de détention de leurs fils, tous trois membres du PKK. Puis, en 1990 et 1992, deux jeunes sympathisantes du parti, Zekiye Alkan et Rahsan Demirel, s'immolent par le feu à Diyarbakir et Izmir pour protester contre la répression des Kurdes. Enfin, en 1992, une jeune combattante, Gülnaz Karatas, se jette dans le vide pour ne pas être capturée vivante par des soldats turcs. Ce sont ces martyrs héroïques - auxquels il faudrait ajouter certains proches du Serok ("président») assassinés ou tués au combat - qui vont progressivement participer à la construction d'un système qui fait de l'engagement et de l'investissement de soi la seule fin convenable.

Témoins et modèles, ces martyrs sacrificiels (fedai sehitler) vont en effet permettre de donner sens à la souffrance en l'historicisant et de construire un groupe idéal sur la base d'une inversion de cette souffrance. C'est en suivant ces guides, en vouant tout son être au parti et à la figure qui l'incarne que l'on peut devenir un "homme nouveau ", désaliéné de la domination turque et digne du sacrifice des fondateurs, des ancêtres et des héros qui combattirent pour le Kurdistan. Dans l'iconographie du parti, le corps de chaque martyr n'est jamais blessé, mutilé ou calciné : seul le visage vierge du "futur » martyr apparaît ${ }^{18}$. Les martyrs-combattants peuvent également être représentés, en treillis ou en civil, armés d'une Kalachnikov ou non, aux côtés d'Abdullah Öcalan. L'image du corps discipliné et souriant, sur lequel les stigmates de la violence subie n'apparaissent pas encore mais sont déjà inscrits et n'ont plus qu'à se révéler dans la 
mort, est une marque du dévouement à la cause et au chef. Si les ouvrages et les Analyses (Çözümlemeler) ${ }^{19}$ d'Öcalan ne fournissent le plus souvent aucune direction tactique ou stratégique aux militants, ils décrivent sa vie, sa perception du monde et la manière de devenir un «vrai » Kurde. Les jeunes Kurdes de Turquie et d'Europe doivent alors calquer leur vie, leur temporalité, leurs expériences et leurs espoirs sur ceux d'Apo (oncle) et des martyrs emblématiques qui ont fusionné avec lui ${ }^{20}$. Mais il est impossible de savoir si la voie empruntée est juste et si les progrès faits sont suffisants : chercher à être digne de l'Önderlik (la Direction), conserver un esprit de sacrifice qui seul peut témoigner de l'amour porté au chef et tenter de changer son ancienne personnalité asservie à la Turquie passe donc par des techniques de soi individuelles.

Cet idéal d'engagement se concrétise en effet dans les pratiques quotidiennes. Rupture de tous les liens sociaux et familiaux antérieurs, tenue vestimentaire strictement codifiée, hygiène corporelle tout aussi stricte (interdiction d'avoir des relations sexuelles, de boire de l'alcool et - dans la guérilla - de fumer du tabac), manières normées de saluer et de parler, utilisation du seul vocabulaire autorisé, rituels d'allégeance au Lider... La disciplinarisation du corps est l'unique moyen de le soustraire à la domination de l'Etat, de le "kurdifier ». La participation active aux grèves de la faim organisées par le parti en Europe est également une manière de montrer, à la fois au monde et au chef, la vérité de son engagement et la transformation de sa personnalité en vue de devenir un "vrai » Kurde. La pression est parfois encore plus forte sur les femmes, insérées dans un système idéologique qui les place au centre de la lutte nationale ${ }^{21}$.

C'est dans la guérilla et dans les prisons turques que l'emprise de l'institution partisane est la plus forte : écriture de rapports, auto-critique, contrôle mutuel dans un contexte de promiscuité et de danger permanent. L'Europe vient ensuite, avec son réseau d'associations affiliées au parti, implantées dans tous les quartiers kurdes des grandes métropoles, ses réunions et ses mobilisations collectives régulières. Tous les témoignages recueillis tendent à montrer qu'il est finalement beaucoup plus aisé de conserver son autonomie dans les métropoles turques d'une part, où le parti a parfois eu du mal à s'implanter et où l'anonymat et l'insuffisance des moyens de communication ne permet pas une homogénéisation des pratiques et des ethos militants, et dans les régions kurdes non contrôlées par la guérilla d'autre part, où les normes des réseaux sociaux traditionnels peuvent entrer en compétition avec celles édictées par le parti.

Au total, les militants semblent donc disposés - à différents degrés - à se faire violence pour Öcalan et pour la cause : le régime de preuve qui se met en place dès le début des années 1980 tend en effet à ne percevoir la vérité, la fidélité et la sincérité que dans les marques de la discipline et de la violence subie. Pour autant, la mise en lumière de ces mécanismes ne permet ni d'expliquer les processus individuels et collectifs qui conduisent à passer de techniques de soi quotidiennes et routinisées à des pratiques auto-destructrices, ni de comprendre les formes différenciées que prennent ces violences contre soi, et ce d'autant plus que le parti ne peut encourager tous les militants à se supprimer volontairement.

Temps et espaces de l'engagement

16 Si l'idéal d'engagement requis est assez stable dans le temps à partir du début des années 1990, l'investissement quotidien est soumis à des contraintes beaucoup plus fluctuantes, liées parfois aux dissensions internes au parti, mais surtout aux évolutions 
générales du conflit. La rhétorique interne du PKK, fondée à la fois sur l'espoir de succès proches («la victoire est pour demain ») et sur la critique des causes ayant conduit à des défaites ("des éléments féodaux subsistent au sein du parti »), fait en effet peser sur les membres du mouvement l'échec de la stratégie mise en œuvre, qui ne peut être imputable à Öcalan : par manque de foi ou d'engagement, ce sont bien les militants qui sont à l'origine des revers politiques et militaires.

Or, ici comme ailleurs, les violences sacrificielles apparaissent le plus souvent lorsque le parti subit des déconvenues, qui rappellent aux militants l'effectivité de la domination turque et de la répression analysées plus haut. C'est d'abord le cas après la fin du cessez-le-feu unilatéral décrété par le PKK mais ignoré par la Turquie le 8 juin 1993 : pour la première fois en Europe, six femmes sont empêchées de s'immoler par le feu en Suisse et en Allemagne, un mois tout juste après la reprise des hostilités. Puis, quelques mois après l'interdiction du PKK en Allemagne, douze personnes tentent de s'immoler en mars 1994 dans différentes villes allemandes : deux jeunes femmes, Bedriye Tas et Nilgün Yildirim, trouveront la mort et deviendront des "martyres emblématiques " dont les noms seront mentionnés dans presque tous les testaments de martyrs sacrificiels qui suivront. On retrouve ce même phénomène à partir de 1996. La question de l'efficacité des opérations militaires est centrale lors du Ve congrès du Parti (janvier 1995) et Öcalan finit par déclarer un nouveau cessez-le-feu unilatéral en décembre de la même année. Les autorités turques persistant à refuser toute négociation politique, l'idée d'organiser des opérations similaires à celle du Hamas semble avoir été lancée à la IVe conférence du PKK en mars 1996, et va se concrétiser après la reprise des hostilités (10 juin 1996): trois guérilleros se font exploser ${ }^{22}$ et sept personnes s'immolent par le feu entre juin et octobre 1996. La première personne à conduire une attaque-suicide, Zeynep Kinaci, deviendra la combattante la plus célébrée de tous les martyrs sacrificiels. Enfin, les pressions turques sur la Syrie et le départ d'Öcalan en octobre 1998, puis l'arrestation et la condamnation à mort du chef du PKK en février et juin 1999 provoquent la plus grande vague de violences sacrificielles de l'histoire du mouvement : l'» affaire Öcalan » verra quatre-vingt cinq tentatives d'immolation par le feu et au moins dix-huit tentatives d'attaque-suicide (dont au moins douze effectives).

La disjonction soudaine entre l'idéal de la lutte et la réalité du conflit semble donc permettre d'expliquer - au moins en partie - comment un certain nombre de militants mobilisent, dans des situations souvent angoissantes (manifestations, campagne d'attaques de l'armée turque), des figures héroïques qui vont les inciter à passer à l'action. Mais c'est bien le contexte de l'engagement qui va déterminer la forme que prend cette action. La transnationalisation progressive de l'organisation et la multiplication des contraintes qui l'accompagnent ont en effet engendré une différenciation des rôles et une spécialisation des tâches au sein du mouvement. L'analyse de la répartition géographique des immolations par le feu est alors éclairante : $53 \%$ des immolations par le feu de militants ou sympathisants du parti ont eu lieu dans des prisons turques, $30 \%$ en Europe et seulement $16 \%$ dans les régions kurdes et turques hors milieu carcéral. Au-delà, et bien que leur nombre très élevé empêche une telle analyse, tout porte à croire que la répartition géographique des grèves de la faim serait à peu près similaire. Une telle distribution s'explique évidemment par le contexte socio-politique de l'engagement: dures conditions de détention et rareté des modes d'action disponibles en prison ; sentiment de trahison lié à l'exil, travail d'appel à l'opinion publique mais difficulté à peser directement sur l'évolution du conflit à partir de l'Europe ; conservation des liens sociaux antérieurs et 
possibilité de rejoindre les partis kurdes légaux, la milice ou la guérilla dans les régions turques et kurdes. Mais, si on laisse provisoirement de côté la guérilla où les enjeux et les techniques de combat sont spécifiques, il est remarquable que la répartition géographique des immolations par le feu corresponde aux cercles concentriques d'emprise de l'institution partisane observés plus haut. On peut donc supposer que si le contexte socio-politique contraint fortement les répertoires d'action, le poids de l'institution reste bien la variable essentielle dans l'explication des modalités d'investissement des militants.

19 L'analyse des formes que prend la violence contre soi au sein de l'armée du PKK peut permettre de vérifier cette hypothèse. Les violences auto-infligées sont ici l'exception : seulement deux cas d'immolation par le feu ont été recensés ${ }^{23}$. Par contre, au moins dix-sept attaques-suicides et au moins neuf autres tentatives ont été organisées entre 1996 et 2006. Plus important sans doute, toutes ces attaques ont été conduites par des combattants déjà formés et entrainés aux techniques de guérilla et dès lors disposés à tuer pour la cause : contrairement au cas palestinien par exemple, aucun militant politique n'a donc conduit d'attaque-suicide et les cibles de ces attaques sont toujours restées militaires et/ou gouvernementales ${ }^{24}$. Cette observation tend à mettre en évidence les liens qu'entretiennent ethos professionnel et forme que prend la violence contre soi dans le cas kurde. Pour autant, parler de compétences militaires ne signifie pas, loin s'en faut, accréditer les thèses utilitaristes qui mettent en avant la stratégie militaire du parti. Si l'on considère que ces dix-sept attaques ont fait moins de trente morts, force est à nouveau de constater que les attaques-suicides ont bien essentiellement pour origine l'idéal d'engagement qui s'est institué au sein du parti. Cette affirmation est encore renforcée par le fait que sur les dix-sept attaques-suicides, onze ont été lancées par des femmes (65\%), qui subissent - comme on l'a vu - plus de pressions que les hommes, et ce surtout dans la guérilla ${ }^{25}$. L'image de la femmemartyre ayant été théorisée par Öcalan, les femmes guérilleros du PKK pouvaient dès lors s'engager dans des attaques-suicides, " par amour de l'humanité et de la vie » ${ }^{26}$.

20 L'hypothèse d'une certaine continuité entre violences auto-infligées et violences autosacrificielles a donc permis de mettre en évidence les relations qu'entretenaient imaginaire du corps souffrant, idéal d'engagement et violences contre soi. Au-delà, il a été possible de déterminer plus précisément certaines caractéristiques de ces violences. Elles apparaissent surtout lorsque revers et défaites viennent contredire le mythe de la lutte nationale et leurs formes semblent fortement corrélées au contexte sociopolitique dans lequel les acteurs sont insérés. Parler de violences contre soi n'empêche donc pas de tenter de comprendre le recours différencié à différents modes d'action, comme la grève de la faim, l'immolation par le feu ou l'attaque-suicide. Ces conclusions ne prétendent évidemment pas à une validité générale : le recours parfois peu fréquent aux différentes formes de violence contre soi analysées ici ou la violence de type eschatologique de certains groupes radicaux qui visent parfois davantage la population civile que les cibles militaires démontrent a contrario la spécificité du cas kurde. Certains éléments nous permettent néanmoins de penser que des parallèles pourraient être établis avec d'autres groupes marxistes ou nationalistes comme certaines organisations de la gauche radicale turque, les groupes sikhs, tamouls ou palestiniens, les Moudjahidin du peuple iranien, et même l'IRA.

21 Cette étude laisse néanmoins de nombreux points sans réponse, et notamment la question des dispositions biographiques qui conduisent à ne pas se faire violence. Si 
certaines dispositions sociales et culturelles ont bien été avancées ici pour expliquer le passage à la violence contre soi, elles sont en effet communes à nombre de militants du PKK, qui ne s'engagent pourtant pas dans ce type de pratiques. Une analyse comparée en termes de trajectoire biographique ou de carrière militante s'avérerait ainsi très utile à la compréhension des dynamiques individuelles qui encouragent ou préviennent les violences contre soi. Elle permettrait de mieux cerner les relations entre identités sociales des acteurs et modalités d'engagement, tout en évitant d'établir une relation mécanique entre les caractéristiques sociales des individus et les modes d'action utilisés. La spécificité du terrain kurde rend néanmoins une telle démarche extrêmement complexe, en raison surtout des processus psychiques individuels qui permettent de se protéger et de se reconstruire après avoir subi des violences.

\section{NOTES}

1.. Notre corpus, qui vise l'exhaustivité sans pour autant la revendiquer, a été constitué à partir de la presse germanophone et turcophone du parti, des rapports des organisations turques des droits de l'Homme, de la base de données Lexis-Nexis, et à partir de diverses coupures de presse et dépêches d'agences en français, anglais, allemand et turc. Seules les immolations effectives - c'est-à-dire marquées par un début de passage à l'acte - ont été recensées (les menaces d'immolations ont été écartées). $\mathrm{Ne}$ sont cependant pas comptabilisées ici les tentatives empêchées (14 cas sur 178). 2.. Au cours de sa recherche, Johanna Siméant, auteur d'un ouvrage sur les grèves de la faim de sans-papiers (La Cause des sans-papiers, Paris, Presses de Sciences Po, 1998), a, non sans raison, reformulé le titre de sa thèse, qui s'intitulait à l'origine « Les stratégies du sacrifice ». Voir « Brûler ses vaisseaux (sur la grève de la faim). Entretien avec Johanna Siméant », Vacarme, ${ }^{\circ}{ }^{18}$, janvier 2002, http://www.vacarme.eu.org/ article229.html.

3.. D'autres formes d'auto-mutilation sont également possibles, comme se coudre les lèvres, ce qui ajoute de facto une " grève de la soif » à la grève de la faim déjà entamée. Voir par exemple « Ready to die: the asylum-seeker who said he fled torture and was driven to self-mutilation in Britain ", The Independant, 28 mai 2003.

4.. Traduction de « scopic regime ». Voir Feldman A., « Violence and vision. The prosthetics and aesthetics of terror », in Das V., Kleinman A., Ramphele M., Reynolds P. (dirs.), Violence and Subjectivity, Berkeley/Los Angeles, University of California Press, 2000, p. 49 ; Feldman A., Formations of Violence. The Narrative of the Body and Political Terror in Northern Ireland, Chicago, University of Chicago Press, 1991.

5.. Sur la pilosité comme marqueur de l'identité politique, voir Fliche B., "Quand cela tient à un cheveu. Pilosité et identité chez les Turcs de Strasbourg ", Terrain, n³5, 2000, pp. 155-165.

6.. Les photographies, transmises par un soldat turc, ne seront publiées que l'année suivante. Voir « Pictures that will shock the world», The European, 11 janvier 1996 et le film de K. Öz (Fotograf, Yapim 13, 2001) qui se fonde sur cet événement. Contre les ordres de leur direction, des combattants kurdes ont également parfois mutilé des 
soldats turcs. Voir Flach, A., Jiyanekê din - ein anderes Leben. Zwei Jahre bei der kurdischen Frauenarmee, Köln, Mezopotamien Verlag, 2003, p. 100.

7.. Siméant J., « La violence d'un répertoire. Les sans-papiers en grève de la faim », Cultures \& Conflits, n 9-10, Paris, L'Harmattan, 1993.

8.. Johanna Siméant note le rôle majeur qu'ont pu jouer Turcs et Kurdes dans les grèves de la faim de sans-papiers en France, au point d'institutionnaliser les grèves au thé sucré pour tenir plus longtemps, alors même que les médecins considèrent cette technique comme plus dangereuse pour les grévistes (Siméant J., La Cause des sanspapiers, op. cit., p. 302).

9.. Ibid., p. 307.

10.. Bozarslan H., «Quelques remarques sur le discours historiographique kurde en Turquie : 1919-1980», Asien Afrika Lateinamerika, vol. 49, 2001, pp. 47-71.

11.. Pour un exemple récent, voir par exemple Kon-Kurd, You are Responsible for this Genocide! Bu Soykirim Sorumlulugundan Kaçamazsiniz !, Bruxelles, décembre 1998. Pour des observations similaires dans le cas sikh, voir Axel B.K., The Nation's Tortured Body: Violence, Representation and the Formation of a Sikh " Diaspora ", Durham, N.C., Duke University Press, 2001.

12.. De manière significative, ce mode de dénonciation a été repris par les autorités turques. Voir Effusion de sang en Anatolie, de la terreur à la sauvagerie, Belge/Centre de recherches orientales, Istanbul, 1992 ou « Unutulan fotograflar » [« les photographies oubliées »], album de photographies d'abord diffusé sur le site Internet de l'Etat-major de l'armée turque et aujourd'hui accessible sur : http:// www.pkkgercegi.net/unutulanfotograflar/default.htm. 13.. Goffman E., Stigmate. Les usages sociaux des handicaps, Paris, Minuit, 1975. 14.. Quand les blessures apparaissent, c'est le plus souvent à la manière d'une autopsie, qui est normalement chargée de découvrir la "vérité ». Mais la photographie médicale s'est d'abord intéressée au malade puis à la maladie (passage du malade à la plaie), alors que le PKK semble passé de la blessure au militant, ce qui ne favorise pas le deuil, surtout si les corps n'ont pas de sépulture ou s'ils doivent être enterrés dans la joie. Voir Harding T. et La Harpe R. «L'autopsie : violence ou réparation? », in Porret M. (dir.) Le Corps violenté, du geste à la parole, Genève, Droz, 1998, pp. 287-292. Voir aussi Foucault M., Naissance de la clinique, Paris, PUF, 1963, p. 173.

15.. On peut recenser une quatrième catégorie de martyrs, celle des violences intrakurdes. Voir Bozarslan H., «La figure du martyr chez les Kurdes », in Mayeur-Jaouen, C. (dir.), Saints et héros du Moyen-Orient contemporain, Paris, Maisonneuve et Larose, 2002, pp. 335-347.

16.. Tejel J., « Le Khoyboun et la fabrication des premiers martyrs du nationalisme kurde ", Etudes kurdes, n6, 2004, pp. 41-58. Voir aussi, sur les symboles du nationalisme kurde, Strohmeier M., Crucial Images in the Presentation of a Kurdish National Identity. Heroes and Patriots, Traitors and Foes, Leiden, Brill, 2003. 17.. En 1978, le programme du PKK résume ces révoltes en quelques lignes, et ne mentionne même pas le nom de leurs chefs (PKK, Der Weg der Revolution Kurdistans (Manifest), Wesanen Serxwebûn/Agri Verlag, Köln, 1986, traduction de Kürdistan Devrimin Yolu - Manifesto, 1978). Pour les fondateurs du parti, les «martyrs » exemplaires sont davantage les dirigeants de la gauche radicale turque, exécutés en 1972 et 1973.

18.. Excepté au tout début des années 1980 , avec notamment des photographies de Mazlum Dogan torturé. Voir Kurdistan Report, n , novembre 1982 et n 3, mai 1983. 
19.. Les Analyses d'Öcalan sont de longs discours publiés sous forme de cassettes vidéo puis d'ouvrages qui servent de base à la formation des militants et sont le préalable à leur auto-critique.

20.. Bozarslan $\mathrm{H}$., « Le nationalisme kurde, de la violence politique au suicide sacrificiel », Critique internationale, $\mathrm{n}^{\circ} 21$, octobre 2003, pp. 93-115.

21.. En un mot, c'est le matriarcat, seul système en harmonie avec la nature, qui symbolisait la société kurde traditionnelle en Mésopotamie. Le patriarcat n'arrive qu'avec la colonisation turque, persane et arabe : les femmes perdant leur pouvoir, c'est tout le peuple kurde qui devient opprimé. La libération du Kurdistan ne peut ainsi passer que par la libération préalable de la femme, qui devient la clé du mouvement national. Et c'est à la femme elle-même, dont la faiblesse et la trahison potentielle sont les causes de l'aliénation, qu'il revient d'oeuvrer le plus à cette libération : par la transmission de la culture kurde à ses enfants, par la lutte armée et par des actes " extraordinaires ", à l'image des « déesses kurdes » de l'âge d'or. Voir Öcalan, A., Kadin ve Aile Sorunu [ « La question de la femme et de la famille »], Istanbul, Melsa, 1992 et PJA, Partei der Freien Frau. Programm, Lieu de publication inconnu (Allemagne), Jina Serbilind Verlag, 2003 (1re édition en turc en 2002).

22.. Trois tentatives d'attaques-suicides pourraient également avoir été empêchées par les services de sécurité turcs. Selon des sources gouvernementales turques, le cessez-lefeu devait d'ailleurs être rompu par une attaque-suicide le 10 juin 1996, mais la militante a été arrêtée avant de pouvoir passer à l'action.

23.. Le premier cas - controversé - correspond au suicide ou à l'assassinat d'une dissidente en août 2000 ; le second cas a été recensé en février 2006, soit quelques semaines avant la reprise des affrontements systématiques entre l'armée du PKK et les forces turques.

24.. Ces attaques feront néanmoins un certain nombre de victimes civiles.

25.. Cette remarque ne doit pas laisser penser que le taux de féminisation des immolations par le feu obéit également aux cercles concentriques d'emprise de l'institution partisane mis en évidence plus haut. La question est en fait plus complexe : la place des femmes est variable dans les différentes branches du parti et l'immolation par le feu est une technique de suicide (non politique) relativement répandue au Moyen-Orient, notamment chez les femmes.

26.. Voir la lettre qu'a adressée Zeynep Kinaci (Zilan), auteur du premier attentatsuicide du PKK, à Abdullah Öcalan : Özgür Kadin Hareketi Sehitler Albümü [Album des martyrs du Mouvement de la femme libre], Jina Serbilind, Lieu de publication inconnu (Allemagne), 2005, p. 26.

\section{RÉSUMÉS}

Parler de violences contre soi, c'est faire l'hypothèse d'une continuité relative entre violences auto-infligées (les grèves de la faim par exemple) et violences auto-sacrificielles (les « attaquessuicides »). Le développement, au sein du conflit kurde, d'un certain imaginaire du corps souffrant a en effet conduit le Parti des travailleurs du Kurdistan (PKK) à renverser ce stigmate et 
à construire un système au sein duquel seuls l'investissement de soi et la discipline permettaient d'être à la hauteur des exigences du chef. C'est la fabrique des martyrs et la diffusion d'un certain idéal d'engagement qui a permis aux membres et sympathisants du parti de recourir, en fonction des circonstances, à telle ou telle forme de violence contre soi.

To speak of violence against oneself is to posit the hypothesis of a relative continuity between self-inflicted violence (for example hunger strikes) and self-sacrificial violence (the so called "suicide attacks"). The development, throughout the Kurdish conflict, of an imaginary of the suffering body drove the Kurdistan Workers Party to reverse this stigma and to conceive a system in which only self-commitment and discipline allowed to live up to the demands of the leader. It is the construction of the martyr and the diffusion of a particular ideal of engagement that has allowed the members and sympathisers of the party to resort, depending on the circumstances, to different forms of "violence against oneself".

\section{INDEX}

Mots-clés : guérillas et organisations clandestines, kamikaze (missions suicide), mouvements politiques, séparatismes/ autonomismes

Thèmes : PKK

Index géographique : Irak, Kurdistan, Moyen-Orient, Turquie

\section{AUTEUR}

\section{OLIVIER GROJEAN}

Doctorant à l'EHESS, Olivier Grojean a enseigné la sociologie politique à l'université de Lille-2. Il a notamment publié « Les répertoires du conflit kurde », in Dorronsoro G. (dir.), La Turquie conteste. Mobilisations sociales et régime sécuritaire, CNRS Editions, 2005, pp. 167-182. 